\title{
Neuromyelitis optica spectrum disorder with delayed lesion on spinal cord magnetic resonance imaging
}

\author{
Ji Eun Han, Hye Rin Yoon, Joong hyun Park, Jeong Yeon Kim*
}

* Correspondence

Jeong Yeon Kim, MD, PhD

Department of Neurology,

Inje University, Sanggye Paik Hospital

1342 dongil- ro, Nowon-gu, Seoul, Korea

Tel: +82-2-950-1090

jykimmd@paik.ac.kr

\begin{abstract}
Introduction
The term Neuromyelitis optica spectrum disorder (NMOSD) is a recently proposed for neuromyelitis optica and related syndromes. Along with serum anti-aquaporin 4 antibody status, detection of a lesions extending over $\geq 3$ contiguous spinal cord segments (longitudinally extensive transverse myelitis, LETM) associated with acute myelitis is the most specific neuroimaging characteristic of NMOSD. 1 We present a case of NMOSD with delayed lesion on spinal cord magnetic resonance imaging.
\end{abstract}

\section{Case Report}

A 36-year-women came to our emergency department with a 2-day history of inability to walk and voiding difficulty. She felt a weakness of both legs during stretching, and did not have lower back pain. She had no recent history of infection and vaccination. She reported hospitalization because of a loss of vision in her right eye five years ago. Her visual acuity was 0.1 with reduced color perception, and both pupils showed a poor response to light stimulation. Blood pressure was $130 / 90 \mathrm{mmHg}$, pulse was regular with 94 beats per minute and respirations 12 breaths per minute. There were no skin lesions, arthralgia eyeball pain and photosensivity. Neurologic examination revealed diminished motor strength in lower extremities with Medical Research Council (MRC) grade $2 / 2$ in hip flexion/extension, knee flexion/extension, ankle dorsi/plantarflexion, and foot inversion. There was hyperesthesia below the T10 level, bilateral positive Babinski sign and ankle clonus, and increased deep tendon reflexes on the knee. No neck rigidity and cranial nerves abnormality was noticed. Inflammatory parameters were not elevated and chest $X$ ray was normal. Routine laboratory data was normal, including complete blood count (Hemoglobin $14.1 \mathrm{~g} / \mathrm{dL}$, white blood cell count 7.190/ uL with $81 \%$ neutrophils), renal, liver and thyroid function tests. Cerebrospinal fluid (CSF) analysis revealed pleocytosis (lymphocyte count of $50 / \mathrm{mm} 3$ ) with elevated albumin concentration $(746 \mathrm{mg} / \mathrm{dL}$; normal range 10-30), but otherwise including CSF glucose was normal. CSF oligoclonal band was analyzed with concurrent serum sample and the results were negative. Visual evoked potential test showed no detection of P100 latency from right eye stimuli. Serum anti-aquaporin-4 (anti-AQP-4) antibody test was positive. To exclude other autoimmune diseases, we evaluated autoimmune antibodies in serum. Rheumatoid factor, cantineutrophil cytoplasmic antibodies (ANCA), pANCA, anti-ds DNA, and paraneoplastic antibodies including anti-Hu, anti-Yo, and anti-Ri were negative results. Magnetic Resonance Imaging (MRI) of the brain and thoracic spinal cord was performed the next day after the hospitalization (after 3 days of symptoms onset) to confirm the presence of other differential diseases. No lesions such as lesions in brainstem, incidental or unlocalizable finding of AVF or white matter changes suspicious CNS demyelinating disease were not founded (Figure $1 \mathrm{~A}$, B).

Despite of the normal thoracic spinal MR imaging, presence of clear clinical evidence of thoracic myelopathy and history of optic neuropathy with abnormal evoked potential findings were felt to be more consistent with a neuromyelitis optica or multiple

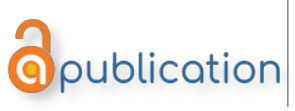


sclerosis, so she was started on a five day course of intravenous methylprednisolone ( $1 \mathrm{~g}$ IV qd for 5 days and then tapered to $1 \mathrm{mg} / \mathrm{kg} / \mathrm{d}$ ) was started. Five days later (after 8 days of symptoms onset), repeat T2-weighted thoracic spine MRI (Fig. 1 C, D) revealed markedly hyperintensity lesion in T2-10 spinal cord with moderate swelling of the cord at these levels. The patient was finally diagnosed Antiaquaporin 4 antibody positive neuromyelitis optica spectrum disorder and she was treated with immunosuppressive treatment (azatioprine $2 \mathrm{mg} / \mathrm{kg}$ daily). She exhibited slowly improvement of weakness with a comprehensive rehabilitation program, although she remained paraplegic and confined to a wheelchair.
Spinal MR imaging with and without contrast is the initial investigation of choice for evaluation of acute myelopathy. 2 In our patient, the initial clinical manifestations indicated a thoracic level suggesting acute transverse myelitis. But, no abnormal findings were observed in the initial MR imaging, and characteristic longitudinally extensive transverse myelitis findings were detected only in the follow up spinal imaging performed 5 days later. We concluded that there was no abnormality in the initial imagimg because lengthy T2-weighted lesion did not developed fully in the few days after clinical symptom onset. Initial negative spinal MR imaging does not exclude neuromyelitis optica spectrum disorder and a follow imaging investigation with closed neurological observation is necessary.
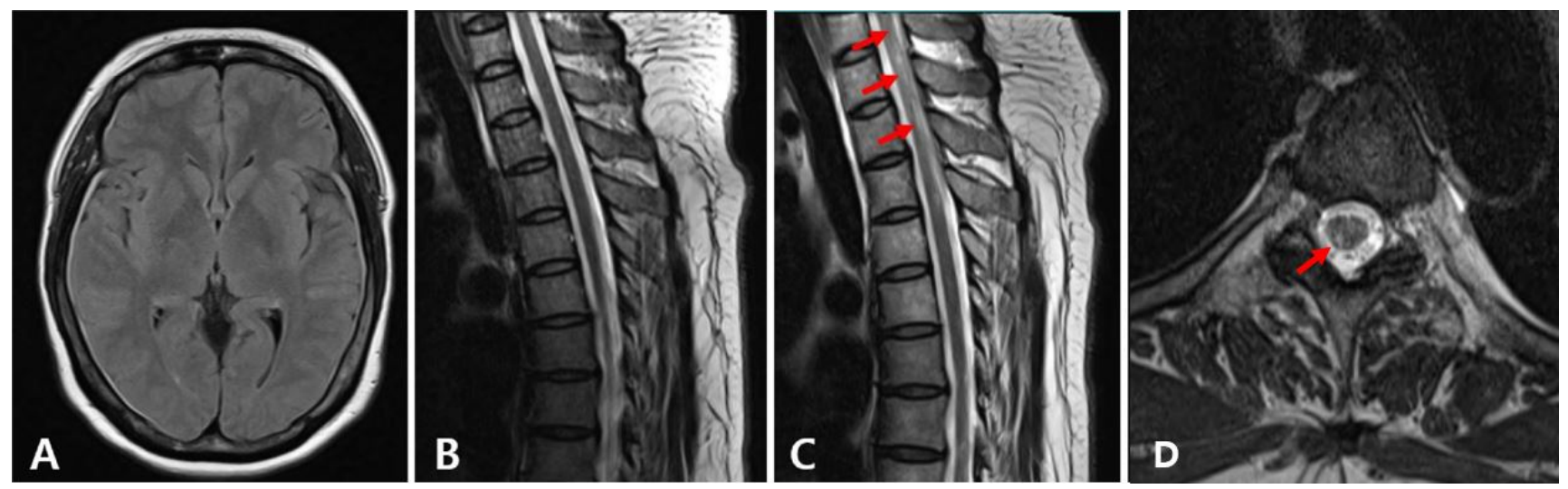

Figure. Brain and spine MRIs of a patient. Axial FLAIR image of brain (A) and sagittal T2- weighted MRI of thoracic spinal cord after 3 days of symptoms onset (B) shows normal brain and spinal cord. Follow up spinal MRI shows longitudinally extensive transverse myelitis (LETM) lesions (red arrows) on thoracic saggital (C) and axial (D) vertebral segments after 8 days of symptoms onset.

\section{Copyrights}

Received: Oct 8, 2018

Accepted: Nov 2, 2018

Published:

\section{Copyrights}

(C2018 Ji Eun Han et al.; licensee OA Journal of Clinical Case Reports. This is an Open Access article distributed under the terms of the Creative Commons Attribution License (http:// creativecommons.org/licenses/by/4.0).

\section{References}

1. Wingerchuk DM, Lennon VA, Pittock SJ, et al. (2006) Revised diagnostic criteria for neuromyelitis optica. Neurology 23: 1485-1489.

2. Wingerchuk DM, Banwell B, Bennett JL, et al. (2015) International consensus diagnostic criteria for neuromyelitis optica spectrum disorders. Neurology 85: 177-189. 